\title{
Elastic Scattering as a Cause of Quantum Dephasing: The Conductance of Two-Dimensional Imperfect Conductors
}

\author{
Yu. V. Tarasov \\ Institute for Radiophysics 8 Electronics, National Academy of Sciences of Ukraine, \\ 12 Acad. Proskura St., Kharkov 61085, Ukraine
}

(November 14, 2018)

\begin{abstract}
A method is proposed for studying wave and particle transport in disordered waveguide systems of dimension higher than unity by means of exact one-dimensionalization of the dynamic equations in the mode representation. As a particular case, the $T=0$ conductance of a two-dimensional quantum wire is calculated, which exhibits ohmic behaviour, with length-dependent conductivity, at any conductor length exceeding the electron quasi-classical mean free path. The unconventional diffusive regime of charge transport is found in the range of conductor lengths where the electrons are commonly considered as localized. In quantum wires with more than one conducting channel, each being identified with the extended waveguide mode, the inter-mode scattering is proven to serve as a phase-breaking mechanism that prevents interference localization without real inelasticity of interaction.
\end{abstract}

PACS numbers: 71.30.+h, 72.15.Rn, 73.50.-h

\section{INTRODUCTION}

Since the original formulation of the localization problem by Andersond, the question of whether electronic states in disordered systems are localized at any strength of disorder or a mobility edge can be defined by a critical level of disorder has become a central issue. In the former case this yields an insulating-type behaviour of large samples, while in the latter the metallic-type transport of conducting electrons is allowed due to the existence of extended states.

It was subsequently ascertained that the answer to this question depends substantially on the dimensionality of the disordered system. For the case of one-dimensional (1D) conductors in the limit of vanishing temperature one can prove in a mathematically rigorous way (see Ref. 2 and references therein) that the spectrum of the electrons, subject to an arbitrarily small but finite random potential in infinitely long samples, is purely discrete, i.e. all electron states are necessarily localized irrespective of their energy. As a result, the DC conductivity vanishes for such systems, whereas in finite 1D samples the conductance falls exponentially as the length of the conductor grows.

In contrast to the 1D case, no mathematically rigorous theory of localization exists for two- (2D) and treedimensional (3D) random systems. Formulation of the problem in terms of the renormalization group 3 (RG) led to the one-parameter scaling hypothesis of localizationt and appeared to provide a considerable progress in studying systems of dimensionality greater than unity. The main conviction gained from this development was that all the electron states in both 1D and 2D disordered systems are localized at arbitrary small disorder. Hence, the metal-insulator transition (MIT) is usually pelieved to occur only in 3D systems, while (sufficiently large) 1D and 2D systems are always Anderson insulators.

This opinion, established quite long ago, has recently been challenged after unexpected experimental detection of MIT in dilute 2D electron and hole systems. 10 Originally obtained on Si-MOSFETs, the results have entailed numerous experimental findings of MIT in other dilute 2D systems 1115 leading to the well-founded belief that the effect is of a rather general nature. To elucidate the unconventional experimental facts, different theoretical approaches were put forward including quite disputable ones (see the discussion in Ref. 16). Among the variety of options the greatest anticipations in explaining the experiments are mostly associated with accounting for the Coulomb interaction of carriers.17. 18 Yet the transport theories for correlated particles in the presence of disorder still cannot claim for general acceptance because of the substantial contrgyersy in the interpretation of the role of interaction within different parameter domains which correspond to diffusive 19 . 20 and localized21 23 regimes.

Traditional understanding of electron localization in 2D disordered systems and numerous experimental facts which indicate the metallic nature of charge transport at low temperatures have brought about active progress in the line of study that is identified with quantum dephasing. The main efforts in this direction are focused on the detection of various phase-breaking mechanisms responsible for the delocalization of quasi-particles primarily localized by disorder. However, in spite of a large body of versions on offer the problem still remains open.

Our intention in this contribution is to re-examine the conventional one-particle approach without appealing to scattering mechanisms other than those characteristic of quenched disorder. Evidence will be given that even within this elementary model the experimental findings of Refs. 10 15, at first sight curious, are as a matter of fact quite 
natural. Numerous attempts to interpret the results of Ref. 10 within the framework of a single-particle approach were made, in particular, by improving the scaling approach. In this study, however, a fundamentally different strategy is chosen which is an alternative to the RG analysis.44 We prefer to obtain the observables directly, while conclusions (though indirect) about the localization of electron states are made on the basis of the results.

It is instructive to recall that working out, even without a profound spectral analysis, practical asymptotic methods for calculating the disorder-averaged many-particle characteristics (conductivity, density-density correlator, etc.) turned out to be more helpful for the establishment of a highly advanced theory of 1D random systems than the development of rigorous mathematical foundations. 25 . 28 The usefulness of such an approach can be attributed to the fact that in the context of the above-mentioned essentially perturbative methods one has managed to trace with the required accuracy the effect of mutual interference of quantum waves corresponding to multiply backscattered current carriers. In such a way, physical results entirely consistent with the anticipations based on mathematical predictions were eventually obtained. The present research was primarily induced by long-standing discontent associated with the lack of arguments of a comparable standard, either in favour of localization or against it, as applied to 2D systems of degenerate electrons subject to a static random potential.

Commonly, the presence of inelastic scattering mechanisms is held responsible as a main cause of preventing quantum interference and, thus, Anderson localization.29 Among these are the electron-phonon and electron-electren interactions and other conceivable methods of energy interchange between the electron bath and the environment. 30 These can lead to the loss of phase (meaning energy) memory or, in other words, phase coherence of electronic states. Note in this connection that in the 1D case the demand of energy coherence admits a large transfer of momentum for onefold scattering of degenerate electrons in the backward direction. This leads inevitably to considerable local breaking of spatial (instead of temporal) phases of the wavefunctions. It was recognized that such a large violation of spatial phases is quite helpful when deriving a constructive theory of $1 \mathrm{D}$ quantum transport. On the basis of such arguments, the selection of efficient subsets of terms in perturbative expansion which are responsible for the effective interference of electronic waves scattered iteratively in a backward direction was suggested.25 This interference finally results in the formation of true localized states, even when one starts their perturbative construction from plain-wavelike trial functions which belong to a mathematical class different from that of localized functions.

An analogous scenario of the perturbative formation of localized states in $2 \mathrm{D}$ systems cannot give the same result, since for sufficiently isotropic scattering the spatial coherence of the wavefunctions is already violated at distances of the order of the quasi-classical mean free path even for weak energy scattering. The coherence is maintained efficiently only within a small phase volume, thefresulting in the relative smallness of the interference corrections, usually known as weak localization corrections 3132 for non-one-dimensional systems.

Nevertheless, the analysis of the problem of multidimensional localization with the use of a one-dimensional scenario turns out to be quite productive. As we shall prove below the problem of electron transport in 2D open system of waveguide type can be reduced without any approximations to the set of one-dimensional (though non-Hermitian) problems for the quantum waves propagating in individual conducting channels. The channels will be identified with extended waveguide modes. For the corresponding dynamic equations that are one-dimensional, an opportunity arises for making substantial use of spatial phases of the wavefunctions instead of their temporal parts. This turns out to be preferable from the technical point of view for solving stationary problems of the electron, as well as classical wave, transport. The reason for the usefulness of such a procedure lies in the fact that in one-dimensional problems spatial averaging has been shown to be highly adwantageous, leading to the reduction of the perturbative expansions of the physical observables to a summable series.25.26

Being exactly quantum in nature, the waveguide approach used here is, to a certain degree, less obvious than semi-classical ones usually applied in most localization theories. Thus, in its context the clarity of the path-based interpretation is substantially lost. At the same time, the benefit of our method is that one-dimensional 'channel' equations enable one to distinguish unambiguously the coherent intra-mode scattering, which is easily interpreted from the standpoint of 1D localization theory, and the inter-mode scattering which corresponds, although not quite directly, to isotropic scattering of semi-classical electrons. The quantum states in different channels are specified by different longitudinal momenta. This difference essentially suppresses interference of primary and scattered electronic waves, if they belong to different channels. As a result, the inter-mode scattering turns out to be an intrinsic origin of the inability for $2 \mathrm{D}$ electrons to be localized by weak static disorder.

The 'one-dimensionalization' procedure suggested in this paper gives an opportunity to highlight the role of spatial coherence in the interference of electronic waves even in a single-particle approximation. 33 The results obtained here by conventional perturbative methods enable us to conclude about the unrealizability of the strong (Anderson) localization in systems whose spatial dimensionality is greater than unity, irrespective of their size. It should be particularly emphasized that decoherence appearing due to inter-mode scattering is unconnected to genuine inelasticity in the interaction of electrons with disorder. However, the difference in longitudinal energy between the conducting channels could be treated as a source of 'hidden inelasticity', if one is accustomed to such an interpretation.

The paper is organized as follows. In the next section, the problem is formulated using linear response theory. 
In section III we develop a method of exact one-dimensionalization which is a central point of the paper. Then, in section IIV, the trial Green functions supremely important for the developed technique are analyzed with the aid of a two-scale perturbation method. A spectral analysis of the electron system is given in section $\mathrm{V}$. In the final two sections we present asymptotic expressions for the conductance and discuss the results. A pair of tedious but important calculations is presented in two appendices.

\section{STATEMENT OF THE PROBLEM}

A common approach used in studies of random systems of various dimensionality is to take a hyper-cube of a certain linear size and vary the size while searching for the conductance scaling. Such an approach seems to be natural when studying spectral properties of closed systems. At the same time, it is not quite appropriate for solving transport problems as applied to structures of waveguide type, in particular, quantum conductors of arbitrary length.

In this paper, we examine the case of a $2 \mathrm{D}$ imperfect rectangular sample of length $L$ in the $x$-direction and width $D$ in the lateral direction $y$. Degenerate non-interacting spinless electrons are assumed to be confined between the hard-wall side boundaries $y= \pm D / 2$, whereas in the direction of current $(x)$ we suppose the system to be open at the strip ends $x= \pm L / 2$. The dimensionless conductance $g(L)$ (in units $e^{2} / \pi \hbar$ ) is calculated directly from linear response theory, 34 whence at zero temperature we have

$$
g(L)=-\frac{4}{L^{2}} \iint d \boldsymbol{r} d \boldsymbol{r}^{\prime} \frac{\partial G\left(\boldsymbol{r}, \boldsymbol{r}^{\prime}\right)}{\partial x} \cdot \frac{\partial G^{*}\left(\boldsymbol{r}, \boldsymbol{r}^{\prime}\right)}{\partial x^{\prime}} .
$$

Here the integration with respect to $\boldsymbol{r}=(x, y)$ is performed over the area occupied by the conductor

$$
x \in(-L / 2, L / 2), \quad y \in(-D / 2, D / 2) .
$$

$G\left(\boldsymbol{r}, \boldsymbol{r}^{\prime}\right)$ is the retarded Green function of the conducting electrons. Within the isotropic Fermi liquid model this function is governed by the equation

$$
\left[\Delta+k_{F}^{2}+i 0-V(\boldsymbol{r})\right] G\left(\boldsymbol{r}, \boldsymbol{r}^{\prime}\right)=\delta\left(\boldsymbol{r}-\boldsymbol{r}^{\prime}\right) .
$$

We adopt the system of units with $\hbar=2 m=1$ ( $m$ is the electron effective mass), so that $\Delta$ is a two-dimensional Laplace operator, $k_{F}$ is the Fermi wavenumber of the electrons, $V(\boldsymbol{r})$ is the 'bulk' static random potential.

The potential $V(\boldsymbol{r})$ in equation (3) will be regarded as a short-range one and not necessarily isotropic. The term 'short-range' implies the characteristic spatial interval over which the potential is substantially varied to be small compared with the 'macroscopic' lengths of the problem, namely the electron mean free path and the conductor length. Being considered as a statistical variable, the potential $V(\boldsymbol{r})$ will be specified by a zero mean value and the binary correlation function

$$
\left\langle V(\boldsymbol{r}) V\left(\boldsymbol{r}^{\prime}\right)\right\rangle=\mathcal{Q} W\left(\boldsymbol{r}-\boldsymbol{r}^{\prime}\right) .
$$

Here the angular brackets denote ensemble averaging and $W(\boldsymbol{r})$ is some function normalized to unity. The explicit form of this function is not so important. In many cases $W(\boldsymbol{r})$ is approximated by the delta-function, $W(\boldsymbol{r})=\delta(\boldsymbol{r})$. However, the method developed in this contribution permits one to consider not only isotropic and not necessarily local scattering events. In addition, the choice of $W(\boldsymbol{r})$ in the form of a delta-function, apart from restricting the physical applicability of the model, is not quite convenient from the technical viewpoint. Thus, for example, when calculating the corrections to the mode spectrum, equations (45), a formal problem can arise of the divergence of the evanescent mode contribution. It is certainly absent provided the potential $W(\boldsymbol{r})$ is not exactly point-supported this problem is familiar in quantum mechanics. 35.36 To get rid of the divergences in the problems of dimension more than one it is merely sufficient to choose the function $W(\boldsymbol{r})$ to be less singular than $\delta(\boldsymbol{r})$. 2 Therefore, in order to focus on the main questions, in place of the correlation equality (4), we shall use the expression below

$$
\left\langle V(\boldsymbol{r}) V\left(\boldsymbol{r}^{\prime}\right)\right\rangle=\mathcal{Q} W\left(x-x^{\prime}\right) \delta\left(y-y^{\prime}\right) .
$$

Additionally, this form of equation (4) allows us to consider anisotropic scattering. Similar to $W(\boldsymbol{r})$ from (4), the function $W(x)$ in (5) is normalized to unity. 


\section{THE ONE-DIMENSIONALIZATION PROCEDURE}

\section{A. The general scheme}

It seems intuitively natural for an open system with the prescribed direction of quasi-particle transport to be considered as to some extent a one-dimensional object. However, the realizability of 'one-dimensionalization' at the level of dynamic equations, which would be quite important from a mathematical perspective, is not $a$ priori apparent. Here the term 'one-dimensionalization' means reduction of the two-dimensional differential equation (3) to a set of one-dimensional equations. Although a system of waveguide type can often be regarded as a collection of one-dimensional quantum channels, the latter are not independent in general. Normally, they are strongly coupled with each other through static or dynamic inhomogeneities present in the problem.

Nevertheless, in what follows we intend to show that just the waveguide nature of a system under consideration enables the mathematical description of the transport problem for a $2 \mathrm{D}$ region (2) to be reduced to a set of independent strictly one-dimensional, although non-Hermitian, problems posed on the interval $x \in(-L / 2, L / 2)$, regardless of the strength of the disorder. To perform the reduction one should merely pass to the mode representation, i.e. Fourier transform in the transverse coordinate $y$, using some complete set $\{\phi(y)\}$ of eigenfunctions of the transverse freeelectron Hamiltonian, namely the Laplace operator in Eq. (3). The conductance (11) acquires the form

$$
g(L)=-\frac{4}{L^{2}} \iint_{L} d x d x^{\prime} \sum_{n, n^{\prime}=1}^{N_{c}} \frac{\partial G_{n n^{\prime}}\left(x, x^{\prime}\right)}{\partial x} \frac{\partial G_{n n^{\prime}}^{*}\left(x, x^{\prime}\right)}{\partial x^{\prime}}
$$

where $N_{c}=\left[k_{F} D / \pi\right]$ is the number of conducting channels or, in other words, extended waveguide modes. Equation (3) is then transformed into a set of coupled ordinary differential equations for the Fourier components $G_{n n^{\prime}}\left(x, x^{\prime}\right)$,

$$
\left[\frac{\partial^{2}}{\partial x^{2}}+k_{n}^{2}+i 0-V_{n}(x)\right] G_{n n^{\prime}}\left(x, x^{\prime}\right)-\sum_{\substack{m=1 \\ m \neq n)}}^{\infty} U_{n m}(x) G_{m n^{\prime}}\left(x, x^{\prime}\right)=\delta_{n n^{\prime}} \delta\left(x-x^{\prime}\right) .
$$

Here $k_{n}^{2}=k_{F}^{2}-(n \pi / D)^{2}$ is the longitudinal energy of the $n$th mode, $U_{n m}(x)$ is the inter-mode matrix element of the potential $V(\boldsymbol{r})$,

$$
U_{n m}(x)=\int_{D} d y \varphi_{n}(y) V(\boldsymbol{r}) \varphi_{m}(y)
$$

Note the difference between the summation limits in Eqs. (6) and (7). Restriction of the summation in (6) by the number of conducting channels implies, strictly speaking, weakness of the electron scattering, to be specified in Sec. IIIB. Under the same assumption formula (1i) itself is valid, where the products of the Green functions of the same kind (both retarded and advanced) have already been omitted. In equation (7), the summation is naturally performed over a complete set of waveguide modes.

Particular attention should be drawn to the fact that the term containing the diagonal (intra-mode) matrix element $U_{n n}(x) \equiv V_{n}(x)$ is initially detached from the sum of Eq. (7), so that the matrix $\left\|U_{n m}\right\|$ hereafter is held off-diagonal in the discrete mode variable. This little technical trick enables one to reduce the problem of finding the overall of the functions $G_{n n^{\prime}}\left(x, x^{\prime}\right)$ to the solution of a subset of purely one-dimensional closed equations for the mode-diagonal functions $G_{n n}\left(x, x^{\prime}\right)$ only. To this end we first introduce the auxiliary trial Green functions $G_{n}^{(V)}\left(x, x^{\prime}\right)(n \in \aleph)$, each obeying the equation

$$
\left[\frac{\partial^{2}}{\partial x^{2}}+k_{n}^{2}+i 0-V_{n}(x)\right] G_{n}^{(V)}\left(x, x^{\prime}\right)=\delta\left(x-x^{\prime}\right)
$$

and Sommerfeld's radiation conditions 3738 at the strip ends $x= \pm L / 2$. These conditions seem to be natural to impose on an open system. For the case of the 1D equation (9) they acquire the form 39

$$
\left.\left(\frac{\partial}{\partial x} \mp i k_{n}\right) G_{n}^{(V)}\left(x, x^{\prime}\right)\right|_{x= \pm L / 2}=0
$$

It implies that the field of the $n$th mode radiated by the point source placed at $x^{\prime} \in(-L / 2, L / 2)$ reaches the corresponding $( \pm)$ end of the interval and then propagates unscattered with the conserved momentum $k_{n}$ beyond the 
ends of the conductor. Possible scattering in the leads attached to the strip from the left and right should be taken into account separately.

Although a solution of the stochastic problem (9) and (10) cannot be obtained in quadratures, in the case of weak scattering specified below by inequalities (20) the main features of the solution can be extracted by due consideration with any desired accuracy in the intra-mode potential $V_{n}(x)$. The necessary analysis is presented in the next section. As for now, we merely consider all the functions $G_{n}^{(V)}\left(x, x^{\prime}\right)$ as a priori known ones whose properties are specified solely by the elastic intra-mode scattering. With these functions chosen as an initial approximation for the exact mode functions $G_{n n}$, only the inter-mode scattering associated with the off-diagonal matrix $U_{n m}(x)$ will then be taken as a perturbation. To implement this intent, we turn from equation (7) to the consequent integral equation,

$$
G_{n n^{\prime}}\left(x, x^{\prime}\right)=G_{n}^{(V)}\left(x, x^{\prime}\right) \delta_{n n^{\prime}}+\sum_{m=1}^{\infty} \int_{L} d x_{1} \mathrm{R}_{n m}\left(x, x_{1}\right) G_{m n^{\prime}}\left(x_{1}, x^{\prime}\right)
$$

Here the kernel

$$
\mathrm{R}_{n m}\left(x, x_{1}\right)=G_{n}^{(V)}\left(x, x_{1}\right) U_{n m}\left(x_{1}\right)
$$

already contains only those harmonics of the potential $V(\boldsymbol{r})$ which are responsible for the inter-mode scattering. A thorough study of system (11) leads to a conjecture that all non-diagonal mode elements $G_{m n}(m \neq n)$ can be expressed only via the diagonal element $G_{n n}$ by means of some linear operator $\hat{\mathrm{K}}$,

$$
G_{m n}\left(x, x^{\prime}\right)=\int_{L} d x_{1} \mathrm{~K}_{m n}\left(x, x_{1}\right) G_{n n}\left(x_{1}, x^{\prime}\right) .
$$

To specify this operator, one should separate the term with the diagonal (in the mode variable) Green function on the right-hand side of equation (11) and substitute all non-diagonal Green functions in the form of equation (13). Then, after renaming the mode variables, we arrive at the following equation for the matrix elements of the operator $\hat{\mathrm{K}}$ :

$$
\mathrm{K}_{m n}\left(x, x^{\prime}\right)=\mathrm{R}_{m n}\left(x, x^{\prime}\right)+\sum_{\substack{k=1 \\ k \neq n}}^{\infty} \int_{L} d x_{1} \mathrm{R}_{m k}\left(x, x_{1}\right) \mathrm{K}_{k n}\left(x_{1}, x^{\prime}\right) .
$$

Equation (14) belongs to a class of multi-channel Lippmann-Schwinger equations that are known to be extremely singular, in contrast to their single-channel counterparts. 10 Nevertheless, by choosing the trial Green function $G_{n}^{(V)}$ as a zero approximation and perturbing it only by the inter-mode potentials, one manages to avoid the above mentioned singularity. Note that mode indices $m$ and $k$ in Eq. (14) take all the positive integer values except for the value $n$. This urges one to interpret the functions appearing in (14) as matrix elements of operators acting in two-dimensional mixed coordinate-mode space $(x, m)$ which does not include the mode $n$ (the notation $\overline{\mathrm{M}}_{\mathrm{n}}$ will be used for that space). The presence in Eq. (14) of the right-hand index $n$, which does not belong to $\overline{\mathrm{M}}_{n}$, can be ensured by introducing the projection operator $\mathbf{P}_{n}$ that will make the mode index of any operator standing next to it (both from the left or right) equal to $n$. With this convention accepted, it follows from equation (14) that the operator $\hat{\mathrm{K}}$ implementing relation (13) has the form

$$
\hat{\mathrm{K}}=(\mathbb{1}-\hat{\mathrm{R}})^{-1} \hat{\mathrm{R}} \mathbf{P}_{n}
$$

Here $\hat{R}$ is a $2 \mathrm{D}$ operator acting on $\bar{M}_{n}$ and is specified by the matrix elements (12).

As for the mathematical correctness of the operator representation (15), it depends on the existence of the inverse operator $(\mathbb{1}-\hat{R})^{-1}$. This point is discussed in Appendix $\mathbb{A}$, where we provide a proof that detachment of the intra-mode potential $V_{n}(x)$ in Eq. (7) prevents the possible singularity.

Thus, equations (13) and (15) reduce the problem of finding the complete Green function $G\left(\boldsymbol{r}, \boldsymbol{r}^{\prime}\right)$ within the 2D region (2) to calculation of its diagonal mode components only. In order to do that it is necessary to put $n^{\prime}=n$ in Eq. (7) and substitute all non-diagonal components $G_{m n}$ from equation (13). As a result, the closed equation for the diagonal component $G_{n n}$ is deduced,

$$
\left[\frac{\partial^{2}}{\partial x^{2}}+k_{n}^{2}+i 0-V_{n}(x)-\hat{\mathcal{T}}_{n}\right] G_{n n}\left(x, x^{\prime}\right)=\delta\left(x-x^{\prime}\right) .
$$


In equation (16), in addition to the local intra-mode potential $V_{n}(x)$, the operator potential $\hat{\mathcal{T}}_{n}$ has arisen,

$$
\hat{\mathcal{T}}_{n}=\mathbf{P}_{n} \hat{\mathcal{U}}(\mathbb{1}-\hat{\mathrm{R}})^{-1} \hat{\mathrm{R}} \mathbf{P}_{n}=\mathbf{P}_{n} \hat{\mathcal{U}}(\mathbb{1}-\hat{\mathrm{R}})^{-1} \mathbf{P}_{n}
$$

Here $\hat{\mathcal{U}}$ is the inter-mode operator specified on $\bar{M}_{n}$ by the matrix elements

$$
\left\langle x, l|\hat{\mathcal{U}}| x^{\prime}, m\right\rangle=U_{l m}(x) \delta\left(x-x^{\prime}\right) .
$$

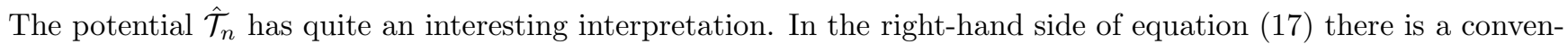
tional $T$-matrix 4014 enveloped by the projective operators, one of which removes the excitation from mode $n$ and the other restores it back to the same mode after the appropriate scattering events within the subspace $\overline{\mathrm{M}}_{\mathrm{n}}$. Hence, the potential $\hat{\mathcal{T}}_{n}$, although corresponding to effectively intra-mode scattering, actually includes all inter-mode scattering events undergone by the excitation while passing over 'closed paths' in the mode space. The intra- and inter-mode scattering mechanisms turn out to be attributed to different potentials in equation (16), facilitating significantly the subsequent interpretation of the results. In what follows the potentials $V_{n}(x)$ and $\hat{\mathcal{T}}_{n}$ will be referred to as those responsible for direct intra-mode and inter-mode scattering, respectively.

Finally, in this subsection we express the conductance (6) through the diagonal elements of the mode Green matrix. After rearranging the terms in Eq. (6) and using relation (13) we obtain

$$
\begin{aligned}
g(L)= & -\frac{4}{L^{2}} \sum_{n=1}^{N_{c}} \iint_{L} d x d x^{\prime}\left[\frac{\partial G_{n n}\left(x, x^{\prime}\right)}{\partial x} \cdot \frac{\partial G_{n n}^{*}\left(x, x^{\prime}\right)}{\partial x^{\prime}}\right. \\
& \left.+\sum_{\substack{m=1 \\
(m \neq n)}}^{N_{c}} \iint_{L} d x_{1} d x_{2} \frac{\partial \mathrm{K}_{m n}\left(x, x_{1}\right)}{\partial x} G_{n n}\left(x_{1}, x^{\prime}\right) \mathrm{K}_{m n}^{*}\left(x, x_{2}\right) \frac{\partial G_{n n}^{*}\left(x_{2}, x^{\prime}\right)}{\partial x^{\prime}}\right] .
\end{aligned}
$$

Expression (19) jointly with equation (16) completes, in principle, the 'one-dimensionalization' procedure introduced at the beginning of this section. In this form the problem under consideration is convenient for a numerical treatment at any disorder strength, because the solution of the 2D problem governed by equation (3) is reduced to a finite set of purely $1 \mathrm{D}$ problems (9) and (16). At the same time, assuming weak electron scattering (in the semi-classical sense), we manage to proceed with our analytical consideration and obtain the results.

\section{B. The weak scattering approximation}

It is natural to specify the intensity of electron scattering in terms of characteristic spatial scales inherent to the problem. Henceforth we recognize the scattering as weak provided the following inequalities hold:

$$
k_{F}^{-1}, r_{c} \ll \ell .
$$

Here $r_{c}$ is the correlation radius of the potential $V(\boldsymbol{r}), \ell=2 k_{F} / \mathcal{Q}$ is the semiclassical mean free path of electrons evaluated within the model of a $\delta$-correlated 2 D random potential, i.e. $W(\boldsymbol{r})=\delta(\boldsymbol{r})$ in Eq. (仼).

Estimation of the norm of the operator $\hat{R}$ specified on $\bar{M}_{n}$ by the matrix elements (12) results in

$$
\|\hat{\mathrm{R}}\|^{2} \sim \frac{D / L}{k_{F} \ell} .
$$

Under conditions (20), this enables us to find an expansion to lowest order in the impurity potential of the operator $\hat{\mathrm{K}}$, Eq. (15), so that it becomes approximately equal to $\hat{\mathrm{R}}$ almost regardless of the conductor aspect ratio. The exact operator $\widehat{\mathcal{T}}_{n}$ from (17) can, in turn, be replaced by the approximate value

$$
\hat{\mathcal{T}}_{n} \approx \mathbf{P}_{n} \hat{\mathcal{U}} \hat{G}^{(V)} \hat{\mathcal{U}} \mathbf{P}_{n}
$$

where the operator $\hat{G}^{(V)}$ is specified on $\overline{\mathrm{M}}_{\mathrm{n}}$ by the matrix elements

$$
\left\langle x, k\left|\hat{G}^{(V)}\right| x^{\prime}, m\right\rangle=\delta_{k m} G_{m}^{(V)}\left(x, x^{\prime}\right) .
$$

Besides the reduction of the $T$-operator (17) to truncated form (22), a substitution of the approximate matrix elements of the operator $\hat{K}$ brings the second term of conductance (19) to the form 


$$
\iint_{L} d x_{1} d x_{2} U_{m n}\left(x_{1}\right) U_{m n}\left(x_{2}\right) \frac{\partial G_{m}^{(V)}\left(x, x_{1}\right)}{\partial x} G_{m}^{(V)^{*}}\left(x, x_{2}\right) G_{n n}\left(x_{1}, x^{\prime}\right) \frac{\partial G_{n n}^{*}\left(x_{2}, x^{\prime}\right)}{\partial x^{\prime}}
$$

which is convenient for performing the ensemble averaging. It will be shown below that all Green functions in (23), and not only the trial ones, may be thought of as independent of the inter-mode potentials $U_{m n}$. Yet correlation of those potentials with the intra-mode one, $V_{n}(x)$, governing the trial Green functions, can be disregarded in view of Eq. (39). As a result, after averaging conductance (19) with the use of (23) and (5), the expression, which will be subject to a further analysis, takes the form

$$
\begin{aligned}
\langle g(L)\rangle= & -\frac{4}{L^{2}} \sum_{n=1}^{N_{c}} \iint_{L} d x d x^{\prime}\left[\left\langle\frac{\partial G_{n n}\left(x, x^{\prime}\right)}{\partial x} \frac{\partial G_{n n}^{*}\left(x, x^{\prime}\right)}{\partial x^{\prime}}\right\rangle\right. \\
& \left.+\frac{\mathcal{Q}}{D} \sum_{\substack{m=1 \\
(m \neq n)}}^{N_{c}} \iint_{L} d x_{1} d x_{2} W\left(x_{1}-x_{2}\right)\left\langle G_{m}^{(V)^{*}}\left(x, x_{2}\right) \frac{\partial}{\partial x} G_{m}^{(V)}\left(x, x_{1}\right)\right\rangle\left\langle G_{n n}\left(x_{1}, x^{\prime}\right) \frac{\partial}{\partial x^{\prime}} G_{n n}{ }^{*}\left(x_{2}, x^{\prime}\right)\right\rangle\right] .
\end{aligned}
$$

\section{ANALYSIS OF THE TRIAL GREEN FUNCTIONS}

The trial Green functions $G_{m}^{(V)}\left(x, x^{\prime}\right)$ enter the potential $\hat{\mathcal{T}}_{n}$, Eq. (22), thus determining the exact mode functions $G_{n n}\left(x, x^{\prime}\right)$, and the second term of the conductance (24). Although these functions appear as subsidiary mathematical objects, they are of great concern for the problem and therefore deserve particular consideration. The study of the trial functions is also instructive, since it may provide useful insights into the analysis of some misinterpretation regarding $2 \mathrm{D}$ localization which existed until recently in the literature.

Note first that the perturbative solution of equation (91) depends substantially on whether the corresponding unperturbed waveguide mode is either extended or evanescent. The Green functions of evanescent modes with $n>N_{c}$ are localized even without any perturbation. To find them in the limit of weak scattering, it is sufficient to restrict oneself to zero-order perturbation in the potential $V_{n}(x)$,

$$
G_{n}^{(V)}\left(x, x^{\prime}\right)=-\frac{1}{2\left|k_{n}\right|} \exp \left(-\left|k_{n}\right|\left|x-x^{\prime}\right|\right), \quad n>N_{c} .
$$

The problem is much more involved for the extended modes, $n<N_{c}$. Inasmuch as the function $G_{n}^{(V)}\left(x, x^{\prime}\right)$ is defined as a solution of the strictly one-dimensional problem (9) and (10), to find it correctly in the context of localization theory the plain-wave-based zero approximation is not quite appropriate. This stems from the fact that in such an approximation it is rather difficult to account for the interference of multiply backscattered waves. Instead we apply the two-scale perturbation method analogous to that used in the theory of non-linear oscillations 42 . This method showed itself well for solving the problem of charge transport in extremely narrow, namely single-mode, surfacecorrugated conducting strips. 43 Below an outline of the method is given together with some essential results. The details of their derivation are deferred to Appendix B.

The method used in Ref. 43 is based on the representation of the 1D Green function, which is the solution of the boundary-value problem (9) and (10), via the solutions of the appropriate Cauchy problems,

$$
G_{n}^{(V)}\left(x, x^{\prime}\right)=\mathcal{W}_{n}^{-1}\left[\psi_{+}(x \mid n) \psi_{-}\left(x^{\prime} \mid n\right) \Theta\left(x-x^{\prime}\right)+\psi_{+}\left(x^{\prime} \mid n\right) \psi_{-}(x \mid n) \Theta\left(x^{\prime}-x\right)\right],
$$

Here the functions $\psi_{ \pm}(x \mid n)$ are linearly independent solutions of the homogeneous equation (9) with the radiation conditions analogous to (10) satisfied at the strip ends $x= \pm L / 2$, according to the 'sign' index. The Wronskian of those functions is $\mathcal{W}_{n}, \Theta(x)$ is Heaviside's step function. This reformulation of a boundary-value problem in terms of an initial-value problem will allow one to perform averaging over the disorder later on. 39

It is advantageous to represent the functions $\psi_{ \pm}(x \mid n)$ as superpositions of modulated harmonic waves propagating in opposite directions along the $x$-axis,

$$
\psi_{ \pm}(x \mid n)=\pi_{ \pm}(x \mid n) \exp \left( \pm i k_{n} x\right)-i \gamma_{ \pm}(x \mid n) \exp \left(\mp i k_{n} x\right) .
$$

Within the framework of the weak scattering approximation (20), the amplitudes $\pi_{ \pm}(x \mid n)$ and $\gamma_{ \pm}(x \mid n)$ vary slowly as compared with the 'fast' exponentials $\exp \left( \pm i k_{n} x\right)$, so that the radiation conditions for $\psi_{ \pm}(x \mid n)$ are reformulated as the 'initial' conditions for the smooth amplitudes as follows: 


$$
\pi_{ \pm}( \pm L / 2 \mid n)=1, \quad \gamma_{ \pm}( \pm L / 2 \mid n)=0 .
$$

In view of the smoothness of $\pi_{ \pm}$and $\gamma_{ \pm}$, differential equations for them can be derived by means of averaging the equations for $\psi_{ \pm}(x \mid n)$ over an arbitrary-valued spatial interval intermediate between the 'microscopic' lengths $k_{n}^{-1}$ and $r_{c}$ on the one hand, and the 'macroscopic' lengths on the other hand. Among the latter lengths are the scattering length, to be specified in the course of the solution, and the sample length $L$. For weak scattering, the equations for $\pi_{ \pm}$and $\gamma_{ \pm}$are reduced to the coupled first-order ones,

$$
\begin{aligned}
& \pi_{ \pm}^{\prime}(x \mid n) \pm i \eta_{n}(x) \pi_{ \pm}(x \mid n) \pm \zeta_{n \pm}^{*}(x) \gamma_{ \pm}(x \mid n)=0 \\
& \gamma_{ \pm}^{\prime}(x \mid n) \mp i \eta_{n}(x) \gamma_{ \pm}(x \mid n) \pm \zeta_{n \pm}(x) \pi_{ \pm}(x \mid n)=0 .
\end{aligned}
$$

Here the variable coefficients $\eta_{n}(x)$ and $\zeta_{n \pm}(x)$ are random fields associated with the intra-mode potential $V_{n}(x)$ in the following way:

$$
\eta_{n}(x)=\frac{1}{2 k_{n}} \int_{x-l}^{x+l} \frac{d t}{2 l} V_{n}(t), \quad \zeta_{n \pm}(x)=\frac{1}{2 k_{n}} \int_{x-l}^{x+l} \frac{d t}{2 l} \mathrm{e}^{\mp 2 i k_{n} t} V_{n}(t) .
$$

For the intermediate property of the averaging interval $2 l$, the fields $\eta_{n}(x)$ and $\zeta_{n \pm}(x)$ are, in fact, nothing but the 'narrow' sets of spatial harmonics of the potential $V_{n}(x)$ with the momenta close to zero and $\pm 2 k_{n}$, respectively. The real field $\eta_{n}(x)$ is responsible for the 'forward' scattering, while the complex one $\zeta_{n \pm}(x)$ for the 'backward' scattering of the $n$th waveguide mode.

Under the assumption of weak scattering, only binary correlators of the random potentials govern the majority of statistical characteristics of physical quantities. It was shown in Ref. 43 that only the two correlation functions, $\left\langle\eta_{n}(x) \eta_{n}\left(x^{\prime}\right)\right\rangle$ and $\left\langle\zeta_{n \pm}(x) \zeta_{n \pm}^{*}\left(x^{\prime}\right)\right\rangle$, of modified random fields (30) may be thought of as non-vanishing. Calculation with the use of model (5) readily gives

$$
\begin{aligned}
\left\langle\eta_{n}(x) \eta_{n}\left(x^{\prime}\right)\right\rangle & =\frac{1}{L_{f}^{(V)}(n)} F_{l}\left(x-x^{\prime}\right), \\
\left\langle\zeta_{n \pm}(x) \zeta_{n \pm}^{*}\left(x^{\prime}\right)\right\rangle & =\frac{1}{L_{b}^{(V)}(n)} F_{l}\left(x-x^{\prime}\right) .
\end{aligned}
$$

Here

$$
L_{f}^{(V)}(n)=\frac{2 D}{3 \mathcal{Q}}\left(2 k_{n}\right)^{2} \quad \text { and } \quad L_{b}^{(V)}(n)=\frac{2 D}{3 \mathcal{Q}} \frac{\left(2 k_{n}\right)^{2}}{\widetilde{W}\left(2 k_{n}\right)}
$$

are the forward and backward mode scattering lengths, respectively; $\widetilde{W}(q)$ is the Fourier transform of $W(x)$ from Eq. (5). The function

$$
F_{l}(x)=\int_{-\infty}^{\infty} \frac{d q}{2 \pi} \mathrm{e}^{i q x} \frac{\sin ^{2}(q l)}{(q l)^{2}}=\frac{1}{2 l}\left(1-\frac{|x|}{2 l}\right) \Theta(2 l-|x|)
$$

in Eqs. (31) is sharp at the scale of 'macroscopic' lengths, so it can be regarded as the $\delta$-function in the 'distributional' sense, $F_{l}(x) \rightarrow \delta(x)$.

Equations (29) and correlation relations (31) enable one to obtain the entire statistical information concerning the trial Green function for any extended mode $n$. In Appendix B derivation of all plain moments of that function is presented with the following result:

$$
\begin{aligned}
& \left\langle\left[G_{n}^{(V)}\left(x, x^{\prime}\right)\right]^{\mu}\right\rangle=\left(\frac{-i}{2 k_{n}}\right)^{\mu} \exp \left[i \mu k_{n}\left|x-x^{\prime}\right|-\frac{\mu}{2}\left(\frac{\mu}{L_{f}^{(V)}(n)}+\frac{1}{L_{b}^{(V)}(n)}\right)\left|x-x^{\prime}\right|\right], \\
& \mu \in \aleph .
\end{aligned}
$$

It is noteworthy that from Eqs. (32) we have the following estimate:

$$
L_{f, b}^{(V)}(n) \sim N_{c} \ell \cos ^{2} \vartheta_{n},
$$


where $\vartheta_{n}$ is a 'sliding angle' of the $n$th mode with respect to the $x$-axis, $\left|\sin \vartheta_{n}\right|=n \pi / k_{F} D$. The value of (35) is coincident, in order of magnitude, with the localization length widely believed to be characteristic for multi-mode quasi-one-dimensional (Q1D) waveguide systems (see, e.g., Refs. 44 47). However, in the present theory lengths (32) are nothing but the extinction lengths of the auxiliary excitations that are not subjected to inter-mode scattering.

Besides plain moments (34), important characteristics of the random function $G_{n}^{(V)}\left(x, x^{\prime}\right)$ are the mixed moments $\left\langle\left[G_{n}^{(V)}\left(x, x^{\prime}\right)\right]^{\mu}\left[G_{n}^{(V)^{*}}\left(x, x^{\prime}\right)\right]^{\nu}\right\rangle$. At $\mu=\nu$ all of them are smooth (not oscillatory) functions of the argument $\left|x-x^{\prime}\right|$ whose spatial decrease is determined by the one-dimensional localization length $\xi_{n}=4 L_{b}^{(V)}(n)$. 目 The second term in (24) contains one of the simplest correlators of this type, the 'density-current' correlator $\left\langle G^{*} \nabla G\right\rangle$. It was already studied in applications to the problem of classical wave transport. 18 We do not present here the exact expression for this correlator since only the fact of its exponential decrease at the localization length $\xi_{n}$ is of significance for our analysis,

$$
\left\langle G_{m}^{(V)^{*}}\left(x, x^{\prime}\right) \frac{\partial}{\partial x} G_{m}^{(V)}\left(x, x^{\prime}\right)\right\rangle \propto \exp \left(-\frac{\left|x-x^{\prime}\right|}{\xi_{n}}\right)
$$

\section{THE MODE STATES SPECTRUM: PERTURBATIVE TREATMENT}

For calculation of the diagonal Green function $G_{n n}\left(x, x^{\prime}\right)$ in equation (16) by means of the perturbation technique, it would make a good sense to reconstruct the operator potential $\hat{\mathcal{T}}_{n}$ so that the mean value equals zero. To that end one has to define the result of the action of the operator $\left\langle\hat{\mathcal{T}}_{n}\right\rangle$ on the function $G_{n n}$. For $n>N_{c}$ (evanescent modes) the function $G_{n n}$ can be left in its unperturbed form (25) due to the weakness of scattering. For $n<N_{c}$, with the operator nature of the potential $\left\langle\hat{\mathcal{T}}_{n}\right\rangle$ and reduced form (22) of the $T$-operator taken into account, the following integral has to be calculated:

$$
\left\langle\hat{\mathcal{T}}_{n}\right\rangle G_{n n}\left(x, x^{\prime}\right)=\int_{L} d x_{1} \boldsymbol{\top}_{n}\left(x, x_{1}\right) G_{n n}\left(x_{1}, x^{\prime}\right)
$$

Here the kernel $\mathrm{T}_{n}\left(x, x_{1}\right)$ is given by

$$
\mathrm{T}_{n}\left(x, x_{1}\right)=\sum_{\substack{m=1 \\(m \neq n)}}^{\infty}\left\langle U_{n m}(x) G_{m}^{(V)}\left(x, x_{1}\right) U_{m n}\left(x_{1}\right)\right\rangle .
$$

On performing the ensemble averaging in Eq. (38), it is justifiable to neglect the correlation between the inter-mode potentials $U_{n m}(x)$ and the intra-mode ones, $V_{m}(x)$. Within the model of point-like scatterers such correlation is entirely absent. Yet even in the case of disorder specified by correlation function (5) it is not difficult, using the definition (8) and the hard-wall model of side boundaries, to ascertain the equality

$$
\left\langle U_{n m}(x) V_{m}\left(x^{\prime}\right)\right\rangle=0 .
$$

This allows us to couple the inter-mode potentials in Eq. (38) only with each other, not affecting the trial functions $G_{m}^{(V)}\left(x, x_{1}\right)$. The averaging procedure thus makes the operator $\left\langle\hat{\mathcal{T}}_{n}\right\rangle$ effectively local.

Since equation (16) is one-dimensional in the space variable, the exact Green function $G_{n n}\left(x, x^{\prime}\right)$ can be represented in a form similar to that used for the trial function $G_{n}^{(V)}\left(x, x^{\prime}\right)$, Eq. (26). Specifically, under weak scattering conditions the function $G_{n n}\left(x, x^{\prime}\right)$, being considered as a function of the first argument $(x)$ only, is composed of two slightly modulated exponential summands of the appearance $\phi_{ \pm}(x)=t_{ \pm}(x) \exp \left( \pm i k_{n} x\right)$. By applying the operator $\left\langle\hat{\mathcal{T}}_{n}\right\rangle$ to the functions $\phi_{ \pm}(x)$ one can factor the smooth amplitudes $t_{ \pm}(x)$ out of the integral thus arriving at the result

$$
\left\langle\hat{\mathcal{T}}_{n}\right\rangle \phi_{ \pm}(x)=\Sigma\left(k_{n}\right) \phi_{ \pm}(x)
$$

where

$$
\Sigma\left(k_{n}\right)=\frac{\mathcal{Q}}{D} \int_{L} d x_{1} W\left(x-x_{1}\right) \exp \left[\mp i k_{n}\left(x-x_{1}\right)\right] \sum_{\substack{m=1 \\ m \neq n)}}^{\infty}\left\langle G_{m}^{(V)}\left(x, x_{1}\right)\right\rangle .
$$


For deriving equation (41), the correlation equality was used

$$
\left\langle U_{n m}(x) U_{k n}\left(x_{1}\right)\right\rangle=\frac{\mathcal{Q}}{D} W\left(x-x_{1}\right) \delta_{m k}
$$

which results from definition (8) and the correlation model (5). The sharp function $W\left(x-x_{1}\right)$ present in Eq. (41) allows us to replace the trial functions $G_{m}^{(V)}\left(x, x_{1}\right)$ by the unperturbed free mode Green functions $G_{m}^{(0)}\left(x, x_{1}\right)$. Then, in the case of even $W(x)$, the factor $\Sigma\left(k_{n}\right)$ acquires the simple form

$$
\Sigma\left(k_{n}\right)=\frac{\mathcal{Q}}{D} \sum_{\substack{m=1 \\(m \neq n)}}^{\infty} \int_{-\infty}^{\infty} \frac{d q}{2 \pi} \widetilde{W}\left(q+k_{n}\right) \widetilde{G}_{m}^{(0)}(q) .
$$

Here $\widetilde{G}_{m}^{(0)}(q)$ is the Fourier transform of the function $G_{m}^{(0)}(x)$, and it is independent of the sign of the momentum $k_{n}$.

From the above analysis it follows that the action of the operator $\left\langle\hat{\mathcal{T}}_{n}\right\rangle$ on the function $G_{n n}\left(x, x^{\prime}\right)$ is reduced to multiplying by, in general, the complex-valued quantity $\Sigma\left(k_{n}\right)$. Using the explicit form of $\widetilde{G}_{m}^{(0)}(q)$,

$$
\widetilde{G}_{m}^{(0)}(q)=\frac{1}{\left(k_{m}+i 0\right)^{2}-q^{2}}
$$

for both real and imaginary parts of the mode 'self-energy' $\Sigma\left(k_{n}\right)=\Delta k_{n}^{2}-i / \tau_{n}^{(\varphi)}$ the expressions are obtained as follows:

$$
\begin{aligned}
\Delta k_{n}^{2} & =\frac{\mathcal{Q}}{D} \sum_{\substack{m=1 \\
(m \neq n)}}^{\infty} \mathcal{P} \int_{-\infty}^{\infty} \frac{d q}{2 \pi} \frac{\widetilde{W}\left(q+k_{n}\right)}{k_{m}^{2}-q^{2}} \\
\frac{1}{\tau_{n}^{(\varphi)}} & =\frac{\mathcal{Q}}{4 D} \sum_{\substack{m=1 \\
(m \neq n)}}^{N c} \frac{1}{k_{m}}\left[\widetilde{W}\left(k_{n}-k_{m}\right)+\widetilde{W}\left(k_{n}+k_{m}\right)\right]
\end{aligned}
$$

The symbol $\mathcal{P}$ in (45a) denotes the principal value. Under the conditions of weak scattering the real part $\Delta k_{n}^{2}$ is always small, $\left|\Delta k_{n}^{2}\right| \ll k_{n}^{2}$, so it can be disregarded without serious consequences. At the same time, 'dissipative' term (45b) plays a crucial role for the further analysis and cannot be omitted. As a result, equation (16) takes the form

$$
\left[\frac{\partial^{2}}{\partial x^{2}}+\kappa_{n}^{2}+i 0-V_{n}(x)-\Delta \hat{\mathcal{T}}_{n}\right] G_{n n}\left(x, x^{\prime}\right)=\delta\left(x-x^{\prime}\right)
$$

where $\kappa_{n}^{2}=k_{n}^{2}+i / \tau_{n}^{(\varphi)}$ and $\Delta \hat{\mathcal{T}}_{n}=\hat{\mathcal{T}}_{n}-\left\langle\hat{\mathcal{T}}_{n}\right\rangle$. Thus, for the analysis of equation 466 we shall regard the set of renormalized energies $\kappa_{n}^{2}(n=1,2, \ldots)$ as representing the new 'unperturbed' spectrum of the system, instead of the original spectrum $\left\{k_{n}^{2}\right\}$. The perturbation theory can now be developed making use of the appropriate zero-mean potentials $V_{n}(x)$ and $\Delta \hat{\mathcal{T}}_{n}$.

Note the difference in summation regions for Eqs. (45a) and (45b). The summation in (45b) turns out to be restricted by the number of conducting channels (extended waveguide modes), because only for $n \leq N_{c}$ the disorderaveraged trial Green functions in Eq. (41) are essentially complex-valued (see Eq. (34) at $\mu=1$ ). The level broadening $1 / \tau_{n}^{(\varphi)}$ implies obligatory presence in the conductor of other extended modes besides the $n$th mode itself. In the case of an extremely narrow strip with $N_{c}=1$ the sum (45b) contains no terms, and thus the system should exhibit true one-dimensional properties. Specifically, the electrons in such systems can be transferred within two regimes only, ballistic apd localized, and the conductance should go down exponentially with the length $L$ exceeding the localization length $\xi_{1} .43$

On increasing the conductor width, as soon as the wire ceases to be single-mode $\left(N_{c} \geq 2\right)$, the situation changes drastically. The $n$ th-mode spectrum acquires the level broadening (45b) and is subjected to both the potentials $V_{n}(x)$ and $\Delta \hat{\mathcal{T}}_{n}$. The physical reason for spectrum 'complexification' with the availability of more than one extended mode in the conductor is actually the randomization of the spatial phase of the electron wavefunction in going between the states with different mode energies and, consequently, mode momenta. It is just the uncertainty of those momenta that destroys the spatial coherence of one-dimensional quantum waves governed by equation (46). This destruction prevents interferential localization of the true mode states of conducting electrons contrary to their trial states. 
To evaluate the 'phase-breaking' effect of the term (45b) note that at any $N_{c}>1$ the estimate $1 / \tau_{n}^{(\varphi)} \sim \mathcal{Q}$ is valid. In particular, in the extreme case of a multimode conductor $\left(N_{c} \gg 1\right)$ with point-like scatterers, replacing the summation in Eq. (45b) by an integration we obtain

$$
1 / \tau_{n}^{(\varphi)} \approx \mathcal{Q} / 4
$$

It is noteworthy that for a large number of conducting sub-bands each level width becomes independent of the mode number $n$ and can thus be thought of as a universal dephasing rate inherent to the 2D conductor in general. Although it is commonly believed that the phase breaking stems from inelastic scattering, the level broadening (45b) has nothing to do with such a scattering mechanism. The only important reason for the existence of an imaginary part of the mode energy is that the conductor has to possess more than one extended mode.

Besides the phase breaking contribution $1 / \tau_{n}^{(\varphi)}$, the role of varying potentials $V_{n}(x)$ and $\Delta \hat{\mathcal{T}}_{n}$ should also be studied. These zero-mean potentials can be assessed through evaluation of the corresponding Born scattering rates $1 / \tau_{n}^{(V)}$ and $1 / \tau_{n}^{(\mathcal{T})}$. Estimation of the operator norms $\left\|\hat{V}_{n}\right\|^{2}$ and $\left\|\Delta \hat{\mathcal{T}}_{n}\right\|^{2}$ yields

$$
\begin{aligned}
& \frac{\tau_{n}^{(V)}}{\tau_{n}^{(\mathcal{T})}} \sim N_{c} \min \left(1, \frac{L}{N_{c} \ell}\right), \\
& \frac{\tau_{n}^{(\varphi)}}{\tau_{n}^{(\mathcal{T})}} \sim \frac{1}{\cos ^{2} \vartheta_{n}} \min \left(1, \frac{L}{N_{c} \ell}\right) .
\end{aligned}
$$

It hence follows that scattering caused by the non-local potential $\Delta \hat{\mathcal{T}}_{n}$ is more efficient than that attributed to the local intra-mode potential $V_{n}(x)$.

On the other hand, the inter-mode scattering caused by the operator potential $\hat{\mathcal{T}}_{n}$ in Eq. (16) is already taken largely into account through the imaginary renormalization of the mode energy in (46). From (48b) it can be seen that the scattering rate $1 / \tau_{n}^{(\mathcal{T})}$ is always small compared to the level width $(45 \mathrm{~b})$ provided that the wire is not extremely long in the $x$-direction, i.e. if the length $L$ does not fall into the interval $L \gg N_{c} \ell$. Yet even within this interval the quantity $1 / \tau_{n}^{(\mathcal{T})}$ cannot exceed the level broadening. If that is the case, the search for strong Anderson localization at any length of the multimode $\left(N_{c} \geq 2\right)$ conducting strip has no sense even without any inelastic scattering mechanisms. Probably, it is necessary to invent some extra conditions to make the interferential localization possible in such conductors, e.g., magnetic field, 19 surface roughness, 50 etc.

\section{CALCULATION OF THE CONDUCTANCE}

Equations (46) and (9) provide a way to perform a direct calculation of the disorder-averaged conductance (24). In this section, to avoid cumbersome expressions the results will be given for the case of a large number of conducting channels, $N_{c} \gg 1$. Nevertheless, all the estimates, as well as the final formulae, are valid for an arbitrary finite number of modes, $N_{c} \gtrsim 1$. In what follows, two summands in equation (24) will be considered separately inasmuch as the calculation techniques and the contribution of these terms in the total conductance differ significantly. The first summand, $\left\langle g^{(1)}(L)\right\rangle$, will be conventionally referred to as the diagonal conductance, since it does not clearly contain any quantities characteristic of the inter-mode scattering. The second term in (24), $\left\langle g^{(2)}(L)\right\rangle$, will be conventionally referred to as the non-diagonal conductance.

Note that the effect of phase breaking, which manifests itself strongly through complexification of the excitation spectrum at $N_{c}>1$, enables one to obtain the solution of equation (46) perturbatively in the potentials $V_{n}$ and $\Delta \hat{\mathcal{T}}_{n}$, i.e. to neglect, in the leading approximation, the interference of quantum waves multiply scattered by those potentials. It follows from estimates (48) that such an approach is absolutely justified for conductors of length $L \ll N_{c} \ell$. Yet even in the case of a Q1D wire of length $L \gg N_{c} \ell$, when the potential $\Delta \hat{\mathcal{T}}_{n}$ in Eq. (46) cannot be disregarded as follows from estimation (48b), the effect of this potential will be shown to be negligibly small.

\section{A. The diagonal conductance}

The presence of the local potential $V_{n}(x)$ in Eq. (46) does not substantially complicate the calculation of the conductance. By applying the method of Ref. 43, this potential can be taken into account with just the same accuracy as was done diagrammatically in Ref. 25. Accounting for this local potential leads to purely interferential corrections, which we are not concerned with in this paper. 
As regards the operator potential $\Delta \hat{\mathcal{T}}_{n}$, to treat it perturbatively keeping in mind the estimate $(48)$ it is advantageous to go over from the differential equation (46) to the consequent integral one,

$$
G_{n n}\left(x, x^{\prime}\right)=G_{n n}^{(0)}\left(x, x^{\prime}\right)+\left(\hat{G}_{n n}^{(0)} \Delta \hat{\mathcal{T}}_{n} \hat{G}_{n n}\right)\left(x, x^{\prime}\right),
$$

Here $G_{n n}^{(0)}\left(x, x^{\prime}\right)$ obeys equation (46) with $\Delta \hat{\mathcal{T}}_{n}=0$. In the leading approximation in the parameter $L / N_{c} \ell \ll 1$, the function $G_{n n}^{(0)}$ has the simple 'unperturbed' form

$$
G_{n n}^{(0)}\left(x, x^{\prime}\right)=\frac{1}{2 i k_{n}} \exp \left\{\left[i k_{n}-1 / l_{n}^{(\varphi)}\right]\left|x-x^{\prime}\right|\right\}
$$

which nonetheless includes most of the inter-mode-scattering effects. In Eq. (50) the mode coherence length $l_{n}^{(\varphi)}$ is associated with the $n$th level broadening (45b), namely $l_{n}^{(\varphi)}=2 k_{n} \tau_{n}^{(\varphi)}$. In the limit $N_{c} \gg 1$ its value equals

$$
l_{n}^{(\varphi)}=4 \ell \cos \vartheta_{n} .
$$

Substitution of the Green function (50) into the first summand of Eq. (24) readily gives the diagonal part of the conductance

$$
\left\langle g^{(1)}(L)\right\rangle=\sum_{n=1}^{N_{c}} \frac{l_{n}^{(\varphi)}}{L}\left[1-\frac{l_{n}^{(\varphi)}}{L} \exp \left(-\frac{L}{l_{n}^{(\varphi)}}\right) \sinh \frac{L}{l_{n}^{(\varphi)}}\right] .
$$

In the limit $N_{c} \gg 1$, by replacing the sum in Eq. (52) with the integral and substituting the coherence length in the form (51), we arrive at the following asymptotic expressions for the conductance (52):

$$
\begin{array}{ll}
L \ll \ell-\left\langle g^{(1)}(L)\right\rangle & \approx N_{c}\left(1-\frac{\pi}{12} \frac{L}{\ell}\right), \\
L \gg \ell \quad-\quad\left\langle g^{(1)}(L)\right\rangle \approx \pi N_{c} \ell / L .
\end{array}
$$

In the limit of $L \gg N_{c} \ell$, it follows from (48b) that the zero-order approximation in $\Delta \hat{\mathcal{T}}_{n}$ is, strictly speaking, insufficient for calculating the function $G_{n n}\left(x, x^{\prime}\right)$. Nevertheless, since the scattering rate $1 / \tau_{n}^{(\mathcal{T})}$ can only be less than or of the order of the level width $1 / \tau_{n}^{(\varphi)}$, the appropriate correction to the conductance can be reasonably estimated by substituting into $g^{(1)}(L)$ the function $G_{n n}\left(x, x^{\prime}\right)$ obtained from (49) to the first order in $\Delta \hat{\mathcal{T}}_{n}$. A simple but tedious calculation brings about the following estimation of the corresponding correction to the conductance:

$$
\left\langle\Delta g^{(1)}(L)\right\rangle \sim\left(\frac{\ell}{L}\right)^{2}
$$

This quantity is apparently small compared with $(53 \mathrm{~b})$. The result in $(54)$ indicates that one may account for the inter-mode scattering in the problem under consideration by means of the phase breaking factor which shows itself only in smearing of the mode energy levels.

\section{B. The non-diagonal term of the conductance}

When calculating the second term $\left\langle g^{(2)}(L)\right\rangle$ in Eq. (24) two essentially different correlators have to be evaluated, the first containing the trial mode Green functions and the second composed of the exact ones. As regards the correlator of the trial functions $G_{m}^{(V)}$, from result (34) it is clear that at $L \ll N_{c} \ell$ those functions can be replaced by the free ones, independently of the mode. Using the function $G_{n n}$ in the form (50) we obtain

$$
\left\langle g^{(2)}(L)\right\rangle=-\frac{\mathcal{Q}}{4 L^{2} D} \sum_{n=1}^{N_{c}} \frac{\left(l_{n}^{(\varphi)}\right)^{3}}{k_{n}} \exp \left(-\frac{L}{l_{n}^{(\varphi)}}\right)\left[\frac{L}{l_{n}^{(\varphi)}} \cosh \frac{L}{l_{n}^{(\varphi)}}-\sinh \frac{L}{l_{n}^{(\varphi)}}\right] \sum_{\substack{m=1 \\(m \neq n)}}^{N_{c}} \frac{1}{k_{m}}
$$

At $N_{c} \gg 1$ this expression is substantially simplified giving the asymptotics as follows: 


$$
\begin{aligned}
& L \ll \ell \quad-\quad\left\langle g^{(2)}(L)\right\rangle \approx-\frac{\pi}{24} N_{c} \frac{L}{\ell}, \\
& \ell \ll L \ll N_{c} \ell \quad-\quad\left\langle g^{(2)}(L)\right\rangle \approx-\pi N_{c} \ell / 2 L .
\end{aligned}
$$

In the case of extremely long conductors with $L \gg N_{c} \ell$, the trial Green functions in (24) cannot be replaced by unperturbed ones so that the effect of 1D localization should be taken into account properly. The 'densitycurrent' correlator standing in (24) was studied in Ref. 48. The result obtained there closely corresponds to analogous results for the 'density-density' and 'current-current' correlators. The main feature of the correlator is that it decays exponentially at the localization length $\xi_{m}=4 L_{b}^{(V)}(m)$. Keeping this in mind, it is not difficult to estimate the non-diagonal term in (24) as

$$
\left\langle g^{(2)}(L)\right\rangle \sim\left(\frac{N_{c} \ell}{L}\right)^{2}
$$

\section{RESULTS AND DISCUSSION}

While comparing results (56) and (57) with those given by equations (53) it can be seen that the non-diagonal part of the conductance is not small relative to the diagonal one only if the conductor length falls within the interval $\ell \ll L \ll N_{c} \ell$. In this case the non-diagonal term is half the size of its diagonal counterpart and has the opposite sign. Hence in the whole range of the conductor length (with the width being kept constant) the conductance is described by the following asymptotic expressions:

$$
\begin{aligned}
\text { (i) } L<\ell: & \langle g(L)\rangle & \approx N_{c} \\
\text { (ii) } \ell \ll L \ll N_{c} \ell: & \langle g(L)\rangle & \approx(\pi / 2) N_{c} \ell / L \gg 1 \\
\text { (iii) } N_{c} \ell \ll L: & \langle g(L)\rangle & \approx \pi N_{c} \ell / L \ll 1 .
\end{aligned}
$$

Strictly speaking, result (58) is valid exactly when the number of channels is large, $N_{c} \gg 1$. Nevertheless, even in the case of $N_{c} \gtrsim 1$ only an insignificant difference occurs, produced by the dependence of the coherence length $l_{n}^{(\varphi)}$ on the mode number $n$. This dependence differs somewhat in the non-semi-classical limit from that given by equation (51), but the difference reduces to a numerical factor of the order of unity only.

The result given by Eq. (58) allows one to distinguish three regimes of charge transport in a quantum conductor, depending on its aspect ratio. Regime (i) corresponds to entirely ballistic transport, both from the semi-classical and quantum points of view. The result obtained exhibits a natural stepwise dependence of the conductance on the transverse size of the wire.

In regimes (ii) and (iii) the semi-classical motion should be regarded as diffusive. This opinion is consistent with the conventional view of classical diffusion since the mean free path $\ell$ is small compared with the sample length $L$, although it can be in arbitrary relation to the conductor width. Furthermore, such an interpretation is supported by the ohmic, i.e. inversely proportional to $L$, dependence of the conductance in both of the indicated regimes. At the same time, it should be particularly emphasized that only in regime (ii), commonly called the weak localization regime, is the result given by the classical kinetic theory reproduced exactly. The expression for the diffusion coefficient in regime (iii) differs from that pertinent to regime (ii) by a factor of two.

Regime (iii) is often called localized, because it is usually supposed that in wires of such a length (commonly referred to as Q1D systems) the Anderson localization should manifest itself to a considerable extent, thus leading to an exponential fall in the conductance. However, from the above analysis it follows that conductors with more than one quantum channel interconnected through scattering mechanisms, even elastic ones, should not exhibit an exponential dependence of kinetic coefficients on the sample length. Such a behaviour is characteristic for the single-mode wires only, which is entirely consistent with theoretical predictions for one-dimensional disordered systems.

To associate findings of this paper with convictions that have prevailed hitherto, it is helpful to examine the electron transport in regimes (ii) and (iii) starting with the trial waveguide states governed by the homogeneous equation (9). Those states are certainly fictitious, they would exist provided the inter-mode scattering were disregarded. If so, the system would indeed represent a set of $N_{c}$ independent one-dimensional conducting channels where the true interferential localization should take place as a result of direct intra-mode scattering from the potentials $V_{n}$. For all of the channels, a hierarchy of localization lengths would exist similar to that representative of equation (35). The length region in (ii) corresponds to the condition when the majority of the trial states are extended. In contrast, 
in regime (iii) all of those states are localized, which is consistent with the expectation of an exponential fall of the conductance with the growth of the sample length.

In reality one certainly cannot disregard the inter-mode scattering in the case of arbitrary quenched disorder. That scattering results in quite strong coupling of the channels or, rather, the trial mode states. The coupling has shown itself through the complexification of true mode spectrum in Eq. (46). Such a complexification suggests that for any extended mode in a multi-mode strip all other extended modes can be thought of as a phase-breaking reservoir destroying quantum interference and hence strong (exponential) localization. Only weak localization corrections due to the local intra-mode potentials can be detected in the both of the diffusive regimes (58). A comprehensive analysis of the matter is beyond the scope of this paper and will be presented elsewhere.

The existence of different diffusion regimes (ii) and (iii) can be interpreted as the dependence of the diffusion coefficient on the conductor aspect ratio. This dependence can hardly be extracted in the framework of the semiclassical approach. It results from the fact that under a gradual transition from regime (ii) to (iii), with the growth of the conductor length, the trial waveguide states undergo sequential localization. This should reduce the probability for the corresponding excitations to leave the conductor through the current terminals, whereas the probability of their scattering into other extended modes should increase. When all the trial states become finally localized, the diffusion coefficient stabilizes at the value corresponding to regime (iii). The dimensionless conductance of such a long wire is less than unity as a consequence of the conventional Ohm's law. It seems that this smallness has previously been the reason to suppose all genuine states in Q1D conductors to be localized.

\section{ACKNOWLEDGMENTS}

The author is grateful to N. M. Makarov for stimulating discussions, A. V. Moroz for help in the interpretation of the results and K. Ilyenko for reading the manuscript.

\section{APPENDIX A: ON REGULARITY OF THE OPERATOR K̂, Eq. (15)}

To ascertain that unity is not among the characteristic numbers of the operator $\hat{R}$, let us take advantage of the operator identity

$$
\log \operatorname{det}(\mathbb{1}-\hat{\mathrm{R}})=\operatorname{Tr} \log (\mathbb{1}-\hat{\mathrm{R}})=\operatorname{Tr} \sum_{k=1}^{\infty} \frac{(-1)^{k}}{k} \hat{\mathrm{R}}^{k}
$$

The last equality in (A1) presumes that the operator norm is limited by $\|\hat{R}\|<1$. This is entirely consistent with the estimate (21).

Consider the traces of the first two terms in sum (A1),

$$
\begin{aligned}
\operatorname{Tr} \hat{\mathrm{R}} & =\sum_{n=1}^{\infty} \int_{L} d x G_{n}^{(V)}(x, x) U_{n n}(x) \\
\operatorname{Tr} \hat{\mathrm{R}}^{2} & =\sum_{n, m=1}^{\infty} \iint_{L} d x d x^{\prime} G_{n}^{(V)}\left(x, x^{\prime}\right) U_{n m}\left(x^{\prime}\right) G_{m}^{(V)}\left(x^{\prime}, x\right) U_{m n}(x) .
\end{aligned}
$$

Since at $n>N_{c}$ the function $G_{n}^{(V)}\left(x, x^{\prime}\right)$ in the case of weak scattering has the form 25), it is easy to conclude that the divergence of the logarithm in (A1) can arise from the first term, given in (A2), provided $U_{n n}(x) \not \equiv 0$. The divergence stems from locality, i.e. coincidence of the arguments, of the Green functions. It manifests itself not only on average but also at a given realization of the random potential entering this term. By separating the diagonal, i.e. intra-mode, potential $V_{n}(x) \equiv U_{n n}(x)$ and making the matrix $\left\|U_{n m}\right\|$ off-diagonal we prevent the singularity of the operator $\hat{\mathrm{K}}$ given by Eq. (15).

\section{APPENDIX B: STATISTICAL MOMENTS OF THE TRIAL GREEN FUNCTIONS}

To perform the ensemble-averaging of the random function $\Phi_{\mu}\left(x, x^{\prime} \mid n\right)=\left[G_{n}^{(V)}\left(x, x^{\prime}\right)\right]^{\mu}, \mu \in \aleph$, in accordance with representation (26) and (27), we first decompose the function $G_{n}^{(V)}\left(x, x^{\prime}\right)$ into the sum of four terms each containing 
narrow packets only of spatial harmonics with phases close to $\pm k_{n}\left(x \pm x^{\prime}\right)$. In doing so one should use the asymptotic expression for the Wronskian $\mathcal{W}_{n}$,

$$
\mathcal{W}_{n}=2 i k_{n}\left[\pi_{+}(x \mid n) \pi_{-}(x \mid n)+\gamma_{+}(x \mid n) \gamma_{-}(x \mid n)\right] .
$$

This results from the assumption of smoothness of the amplitude functions in Eq. (27). Then, after substituting (27) and (B1) into (26), the function $G_{n}^{(V)}\left(x, x^{\prime}\right)$ can be represented in the form of a scalar product

$$
G_{n}^{(V)}\left(x, x^{\prime}\right)=\left(\mathrm{e}^{i k_{n} x} ; \mathrm{e}^{-i k_{n} x}\right)\left(\begin{array}{cc}
\widetilde{G}_{1} & \widetilde{G}_{3} \\
\widetilde{G}_{4} & \widetilde{G}_{2}
\end{array}\right)\left(\begin{array}{c}
\mathrm{e}^{-i k_{n} x^{\prime}} \\
\mathrm{e}^{i k_{n} x^{\prime}}
\end{array}\right)
$$

Here $\widetilde{G}_{i}\left(x, x^{\prime} \mid n\right)$ are the smooth amplitudes constructed from the envelopes $\pi_{ \pm}(x \mid n)$ and $\gamma_{ \pm}(x \mid n)$ as follows:

$$
\begin{aligned}
& \widetilde{G}_{1}\left(x, x^{\prime} \mid n\right)=\frac{-i}{2 k_{n}} A_{n}(x)\left[\Theta_{+} \frac{\pi_{-}\left(x^{\prime} \mid n\right)}{\pi_{-}(x \mid n)}-\Theta_{-} \frac{\gamma_{+}\left(x^{\prime} \mid n\right)}{\pi_{+}(x \mid n)} \Gamma_{-}(x \mid n)\right], \\
& \widetilde{G}_{2}\left(x, x^{\prime} \mid n\right)=\frac{-i}{2 k_{n}} A_{n}(x)\left[\Theta_{-} \frac{\pi_{+}\left(x^{\prime} \mid n\right)}{\pi_{+}(x \mid n)}-\Theta_{+} \Gamma_{+}(x \mid n) \frac{\gamma_{-}\left(x^{\prime} \mid n\right)}{\pi_{-}(x \mid n)}\right], \\
& \widetilde{G}_{3}\left(x, x^{\prime} \mid n\right)=\frac{-1}{2 k_{n}} A_{n}(x)\left[\Theta_{+} \frac{\gamma_{-}\left(x^{\prime} \mid n\right)}{\pi_{-}(x \mid n)}+\Theta_{-} \frac{\pi_{+}\left(x^{\prime} \mid n\right)}{\pi_{+}(x \mid n)} \Gamma_{-}(x \mid n)\right], \\
& \widetilde{G}_{4}\left(x, x^{\prime} \mid n\right)=\frac{-1}{2 k_{n}} A_{n}(x)\left[\Theta_{-} \frac{\gamma_{+}\left(x^{\prime} \mid n\right)}{\pi_{+}(x \mid n)}+\Theta_{+} \Gamma_{+}(x \mid n) \frac{\pi_{-}\left(x^{\prime} \mid n\right)}{\pi_{-}(x \mid n)}\right] .
\end{aligned}
$$

The notation used in Eqs. (B3) is

$$
A_{n}(x)=\left[1+\Gamma_{+}(x \mid n) \Gamma_{-}(x \mid n)\right]^{-1}, \quad \Gamma_{ \pm}(x \mid n)=\frac{\gamma_{ \pm}(x \mid n)}{\pi_{ \pm}(x \mid n)}, \quad \Theta_{ \pm}=\Theta\left[ \pm\left(x-x^{\prime}\right)\right] .
$$

Before averaging the functions (B3) over the random potential, let us note some useful features of the dynamic system (29). Since $\pi_{ \pm}(x \mid n)$ and $\gamma_{ \pm}(x \mid n)$ are the causal functionals of the fields $\eta_{n}(x), \zeta_{n \pm}(x)$ and $\zeta_{n \pm}^{*}(x)$, they are determined by the values of those fields on the intervals $(x, L / 2]$ and $[-L / 2, x)$ for the functionals labelled by the indexes $(+)$ and $(-)$, correspondingly. The Green function elements (B3), which will be subjected to ensemble averaging, are constructed in such a fashion that supports of the random functions entering the functionals of 'plus' and 'minus' type do not meet. Due to the random fields being effectively $\delta$-correlated, see Eqs. (31) and (33), averaging of the functionals with different sign indexes can be performed independently.

It also follows from equations (29) and conditions (28) that all terms of functional series for $\pi_{ \pm}(x \mid n)$ contain an equal number of fields $\zeta_{n \pm}$ and $\zeta_{n \pm}^{*}$, whereas $\gamma_{ \pm}(x \mid n)$ has an extra functional factor $\zeta_{n \pm}$. Since for weak scattering all fields $\eta_{n}(x), \zeta_{n \pm}(x)$ and $\zeta_{n \pm}^{*}(x)$ are approximately Gaussian random processes, only the first summands in square brackets of Eqs. (B3a) and (B3b) remain non-zero after averaging, while the quantities $\left\langle\widetilde{G}_{3,4}\right\rangle$ vanish. By the same arguments, the factor $A_{n}(x)$ in (B3) can be replaced by unity.

In view of the statistical independence of the functions $\eta_{n}(x)$ and $\zeta_{n \pm}(x)$, it is convenient to average over the real field $\eta_{n}(x)$ already at the initial stage. To that end, it is advantageous to perform the following phase transformation of the amplitudes $\pi_{ \pm}$and $\gamma_{ \pm}$:

$$
\begin{aligned}
& \pi_{ \pm}(x \mid n)=\widetilde{\pi}_{ \pm}(x \mid n) \exp \left[ \pm i \int_{x}^{ \pm L / 2} \eta_{n}\left(x_{1}\right) d x_{1}\right] \\
& \gamma_{ \pm}(x \mid n)=\widetilde{\gamma}_{ \pm}(x \mid n) \exp \left[\mp i \int_{x}^{ \pm L / 2} \eta_{n}\left(x_{1}\right) d x_{1}\right] .
\end{aligned}
$$

The new amplitudes $\widetilde{\pi}_{ \pm}$and $\widetilde{\gamma}_{ \pm}$obey the equations

$$
\begin{aligned}
& \widetilde{\pi}_{ \pm}^{\prime}(x \mid n) \pm \widetilde{\zeta}_{n \pm}^{*}(x) \widetilde{\gamma}_{ \pm}(x \mid n)=0, \\
& \widetilde{\gamma}_{ \pm}^{\prime}(x \mid n) \pm \widetilde{\zeta}_{n \pm}(x) \widetilde{\pi}_{ \pm}(x \mid n)=0,
\end{aligned}
$$

where the random field $\widetilde{\zeta}_{n \pm}(x)$ is related to $\zeta_{n \pm}(x)$ by the equality 


$$
\widetilde{\zeta}_{n \pm}(x)=\zeta_{n \pm}(x) \exp \left[ \pm 2 i \int_{x}^{ \pm L / 2} \eta_{n}\left(x_{1}\right) d x_{1}\right]
$$

This latter condition does not modify correlation properties of the backscattering fields, Eqs. (31). Then performing a Fourier transformation of the function $\Phi_{\mu}\left(x, x^{\prime} \mid n\right)$ over $x^{\prime}$, we arrive at the expression conveniently decomposed into the sum of 'plus' and 'minus' functionals,

$$
\widetilde{\Phi}_{\mu}(x, q \mid n)=\left(-\frac{1}{2 k_{n}}\right)^{\mu} \mathrm{e}^{i q x}\left[\widetilde{\Phi}_{\mu}^{(+)}(x, q \mid n)+\widetilde{\Phi}_{\mu}^{(-)}(x, q \mid n)\right] .
$$

Here the functions $\widetilde{\Phi}_{\mu}^{( \pm}$are given by

$$
\widetilde{\Phi}_{\mu}^{( \pm)}(x, q \mid n)= \pm \int_{x}^{ \pm L / 2} d x_{1}\left[\frac{\widetilde{\pi}_{ \pm}\left(x_{1} \mid n\right)}{\widetilde{\pi}_{ \pm}(x \mid n)}\right]^{\mu} \exp \left[-i q\left(x-x_{1}\right)+i \mu k_{n}\left|x-x_{1}\right| \pm i \mu \int_{x_{1}}^{x} \eta_{n}\left(x_{2}\right) d x_{2}\right] .
$$

Averaging functions $(\mathrm{B} 8)$ over the random field $\eta_{n}(x)$ with the use of (31a) readily yields

$$
\widetilde{\Phi}_{\mu}^{( \pm)}(x, q \mid n)= \pm \int_{x}^{ \pm L / 2} d x_{1}\left[\frac{\widetilde{\pi}_{ \pm}\left(x_{1} \mid n\right)}{\widetilde{\pi}_{ \pm}(x \mid n)}\right]^{\mu} \exp \left\{-i q\left(x-x_{1}\right)+\left[i \mu k_{n}-\frac{\mu^{2}}{L_{f}^{(V)}(n)}\right]\left|x-x_{1}\right|\right\} .
$$

To then perform averaging over the fields $\zeta_{n \pm}(x)$ it is convenient to use the dynamic equations for the functions $\widetilde{\Phi}_{\mu}^{( \pm)}(x, q \mid n)$ and $\widetilde{\Gamma}_{ \pm}(x \mid n)=\widetilde{\gamma}_{ \pm}(x \mid n) / \widetilde{\pi}_{ \pm}(x \mid n)$. They read

$$
\begin{gathered}
\mp \frac{d \widetilde{\Phi}_{\mu}^{( \pm)}(x, q \mid n)}{d x}=1-\left[\frac{\mu^{2}}{2 L_{f}^{(V)}(n)}-i \mu k_{n} \mp i q\right] \widetilde{\Phi}_{\mu}^{( \pm)}(x, q \mid n)-\mu \widetilde{\zeta}_{n \pm}^{*}(x) \widetilde{\Gamma}_{ \pm}(x \mid n) \widetilde{\Phi}_{\mu}^{( \pm)}(x, q \mid n), \\
\pm \frac{d \widetilde{\Gamma}_{ \pm}(x \mid n)}{d x}=-\widetilde{\zeta}_{n \pm}(x)+\widetilde{\zeta}_{n \pm}^{*}(x) \widetilde{\Gamma}_{ \pm}^{2}(x \mid n) .
\end{gathered}
$$

These equations stem from definitions (B8) and system (B5) along with the obvious 'initial' conditions

$$
\widetilde{\Phi}_{\mu}^{( \pm)}( \pm L / 2, q \mid n)=0, \quad \widetilde{\Gamma}_{ \pm}( \pm L / 2 \mid n)=0 .
$$

Averaging of (B10) with the use of Furutsu-Novikov formula 39 gives the equation

$$
\frac{d\left\langle\widetilde{\Phi}_{\mu}^{( \pm)}(x, q \mid n)\right\rangle}{d x}=1-\left[\frac{\mu}{2}\left(\frac{\mu}{L_{f}^{(V)}(n)}+\frac{1}{L_{b}^{(V)}(n)}\right)-i \mu k_{n} \mp i q\right]\left\langle\widetilde{\Phi}_{\mu}^{( \pm)}(x, q \mid n)\right\rangle,
$$

from which the result (34) arises immediately.

${ }^{1}$ P. W. Anderson, Phys. Rev. B 109, 1492 (1958).

${ }^{2}$ I. M. Lifshits, S. A. Gredeskul and L. A. Pastur, Introduction to the Theory of Disordered Systems (Wiley, New York, 1988).

${ }^{3}$ F. J. Wegner, Z. Phys. 25, 327 (1976).

${ }^{4}$ E. Abrahams et al., Phys. Rev. Lett. 42, 673 (1979).

${ }^{5}$ Although universality of the one-parameter scaling was quite long challenged attempts were made (and still persist) to improve the scaling approach owing to its relative convenience and simplicity.

${ }^{6}$ A. A. Abrikosov, Solid State Commun. 37, 997 (1981).

${ }^{7}$ V. E. Kravtsov and I. V. Lerner, Solid State Commun. 52, 593 (1984).

8 M. Shreiber and M. Ottomeier, J. Phys.: Condens. Matter 4, 1959 (1992).

${ }^{9}$ V. Dobrosavljević et al., Phys. Rev. Lett. 79, 455 (1997).

${ }^{10}$ S. V. Kravchenko et al., Phys. Rev. B 50, 8039 (1994); Phys. Rev. B 51, 7038 (1995); Phys. Rev. Lett. 77, 4938 (1996). 
${ }^{11}$ D. Popovic, A. B. Fowler, and S. Washburn, Phys. Rev. Lett. 79, 1543 (1997).

${ }^{12}$ P. M. Coleridge et al., Phys. Rev. B 56, R12764 (1997).

${ }^{13}$ M. Y. Simmons et al., Phys. Rev. Lett. 80, 1292 (1998).

14 S. J. Papadakis and M. Shayegan, Phys. Rev. B 57, R15068 (1998).

${ }^{15}$ Y. Hanein et al., Phys. Rev. B 58, R13338 (1998).

${ }^{16}$ I. L. Aleiner, B. L. Altshuler, and M. E. Gershenson, Waves Random Media 9, 201 (1999).

17 D. L. Shepelyansky, Phys. Rev. Lett. 73, 2607 (1994).

18 D. Weinmann, J.-L. Pichard, and Y. Imry, J. Phys. I (France) 7, 1559 (1997).

${ }^{19}$ R. Berkovits and Y. Avishai, Phys. Rev. Lett. 76, 291 (1996).

${ }^{20}$ P. Schmitteckert et al., Phys. Rev. Lett. 80, 560 (1998).

${ }^{21}$ A. L. Efros and F. G. Pikus, Solid State Commun. 96, 183 (1995).

${ }^{22}$ J. Talamantes, M. Pollak, and L. Elam, Europhys. Lett. 35, 511 (1996).

${ }^{23}$ T. Vojta, F. Epperlein, and M. Schreiber, Phys. Rev. Lett. 76, 291 (1996).

${ }^{24}$ S. Ma, Modern Theory of Critical Phenomena (Menlo Park, CA: Benjamin-Cummings, 1976).

${ }^{25}$ V. L. Berezinski, Zh. Eksp. Teor. Fiz. 65, 1251 (1973) [Sov. Phys.-JETP 38, 620 (1974)].

${ }^{26}$ A. A. Abrikosov and I. A. Ryzhkin, Adv. Phys. 27, 147 (1978).

${ }^{27}$ E. A. Kaner and L. V. Chebotarev, Phys. Rep. 150, 179 (1987).

${ }^{28}$ E. A. Kaner and Yu. V. Tarasov, Phys. Rep. 165, 189 (1988).

${ }^{29}$ M. Büttiker, Y. Imry, and R. Landauer, Phys. Lett. 96 A, 365 (1983).

${ }^{30}$ A helpful discussion of the approaches to the problem can be found in P. A. Mello, Y. Imry, and B. Shapiro, LANL preprint cond-mat/9907496.

31 B. L. Altshuler, A. G. Aronov and D. E. Khmelnitski, J. Phys. C: Solid State Phys. 15, 7367 (1982).

${ }^{32}$ B. L. Altshuler, A. G. Aronov, D. E. Khmelnitski, and A. I. Larkin, in Quantum Theory of Solids (Moscow: Mir, 1982) (in Russian).

33 The particular role of spatial phases in quantum mechanics was highlighted as long as the two-slit experiments were explained, see, e.g., R. P. Feynman, R. B. Leighton, and M. Sands, The Feynman Lectures on Physics, Vol. III (Reading, MA: AddisonWesley, 1965)

${ }^{34}$ R. Kubo, J. Phys. Soc. Japan 12, 570 (1957).

${ }^{35}$ A.I. Baz', Ya.B. Zel'dovich, and A.M. Perelomov. Scattering, Reactions, and Decay in Non-Relativistic Quantum Mechanics (Moscow: Nauka, 1971) (in Russian).

${ }^{36}$ L.D. Landau and I.M. Lifshits. Quantum Mechanics: Non-Relativistic Theory (Oxford: Pergamon, 1977).

${ }^{37}$ F.G. Bass and I.M. Fuks, Wave Scattering from Statistically Rough Surfaces (Oxford: Pergamon, 1979).

${ }^{38}$ V.S. Vladimirov, Equations of mathematical physics (Moscow: Nauka, 1967) (in Russian).

${ }^{39}$ V.I. Klyatskin. The invariant Imbedding Method in a Theory of Wave Propagation (Moscow: Nauka, 1986) (in Russian).

40 J. R. Taylor, Scattering Theory. The Quantum Theory on Nonrelativistic Collisions (New York: Wiley, 1972).

${ }^{41}$ R. Newton. Scattering Theory of Waves and Particles (New York: McGraw-Hill, 1968).

${ }^{42}$ N. N. Bogolyubov and Yu. A. Mitropolsky. Asymptotic methods in the theory of non-linear oscillations (Moscow: Nauka, 1974) (in Russian).

43 N. M. Makarov and Yu. V. Tarasov, J.Phys.: Condens. Matter 10, 1523 (1998).

${ }^{44}$ O. N. Dorokhov, Solid State Commun. 51, 381 (1984); Phys. Rev. B 37, 10526 (1988).

${ }^{45}$ P. A. Mello, P. Pereyra, and N. Kumar, Ann. Phys., 181, 290 (1988).

${ }^{46}$ H. Tamura and T. Ando, Phys. Rev. B 44, 1792 (1991).

${ }^{47}$ S.-R. Eric Yang and J. Rammer, Phys. Rev. B 53, 9568 (1996).

${ }^{48}$ V. D. Freylikher and Yu. V. Tarasov, IEEE Trans. Antennas Propag. 39, 197 (1991).

49 A. Malinowski et al., Phys. Rev. Lett. 79, 495 (1997).

${ }^{50}$ A. Garcia-Martin et al., Appl. Phys. Lett., 71, 1912 (1997). 